\title{
Saldırı Tespiti için Makine Öğrenme Yöntemlerinin Karşılaştırmalı Analizi
}

\author{
Mehmet BURUKANLI ${ }^{1}$, Musa ÇIBUK ${ }^{2 *}$, Ümit BUDAK ${ }^{3}$ \\ ${ }^{1}$ Bitlis Eren Üniversitesi, Enformatik Bölümü, Bitlis \\ ${ }^{2}$ Bitlis Eren Üniversitesi, Bilgisayar Mühendisliği Bölümü, Bitlis \\ ${ }^{3}$ Bitlis Eren Üniversitesi, Elektrik-Elektronik Mühendisliği Bölümü, Bitlis \\ (ORCID: 0000-0003-4459-0455) (ORCID: 0000-0001-9028-2221) (ORCID: 0000-0003-4082-383X)
}

\begin{abstract}
$\ddot{O} \mathbf{z}$
İnternet ve ağ teknolojilerindeki hızlı gelişmeler, siber saldırılar ve izinsiz erişimlerin başta olduğu birçok dezavantajı beraberinde getirmektedir. $\mathrm{Bu}$ girişimlerin önceden tespiti, olası saldırıların gerçekleşmeden önlenebilmesini sağlamaktadır. Bu çalışmada makine öğrenme yaklaşımlarının saldırı tespiti üzerindeki performansları araştırılmıştır. Tüm deneyler, açık erişime sunulmuş ve yaygın olarak kullanılan KDD'99 veri kümesi altındaki KDD10CORRECTED ve KDDTEST setleri üzerinde gerçekleştirilmiştir. Sınıflandırıcı olarak, KA, TÖ ve DVM tercih edilmiştir. Veri setleri hem doğrudan sınıflandırıcıların girişi olarak hem de boyut indirgeme tekniği olan TBA uygulanarak sınıflandırılmıştır. Sınıflandırma aşamasında 5-kat çapraz doğrulama tekniği kullanılmıştır. En iyi başarım oranları KDD10CORRECTED veri setinde üzerinde $\% 99,99$ ile Torbalama sinıflandırıcısı, KDDTEST veri setinde üzerinde $\% 97,90$ ile Torbalama siniflandırıcısı, KDD10CORRECTED+KDDTEST veri setinde üzerinde \%100 ile Torbalama sinıflandırıcısı elde etmiştir. Elde edilen sonuçlar kıyaslanarak rapor edilmiştir. Sonuçlar gelecekteki çalışmalar için cesaret vericidir.
\end{abstract}

Anahtar kelimeler: Saldırı tespiti, topluluk öğrenme, destek vektör makinesi, karar ağacı, temel bileşen analizi.

\section{Comparative Analysis of Machine Learning Methods for Intrusion Detection}

\begin{abstract}
Rapid developments of internet and network technologies have brought about many disadvantages, including cyber-attacks and intrusions. The detection of these initiatives beforehand provides the prevention of probable attacks. In this study, the performance of machine learning approaches on intrusion detection has investigated. All experiments have conducted on KDD10CORRECTED and KDDTEST sub-sets of the publicly available KDD'99 dataset. As the classifier, Decision Tree, Ensemble Learning and Support Vector Machine have preferred. Data sets are classified as both directly input of the classifiers and by using Principal Component Analysis, which is a size reduction technique. 5-fold cross-validation technique has used in the classification stage. The best performance rates have been achieved above the KDD10CORRECTED data set with the Bagging classifier with 99.99\%, above the KDDTEST data set with the Bagging classifier with $97.90 \%$, and above the KDD10CORRECTED + KDDTEST data set with the Bagging classifier with $100 \%$. The results have reported by comparing. The results are encouraging for future studies.
\end{abstract}

Keywords: Intrusion detection, ensemble learning, support vector machine, decision tree, principal component analysis.

\section{Giriş}

Son yıllarda internetin yaygın kullanımı, güvenlik tehlikelerini de beraberinde getirmektedir. Özellikle internet üzerinden yapılan e-ticaret uygulamaları ciddi oranda tehlikeli saldırılara maruz kalmaktadır. $\mathrm{Bu}$ saldırılar, kritik iş uygulamalarında iş gücü, zaman ve ürün kaybına yol açarak şirketlerin ciddi anlamda zarara uğratılmasına neden olmaktadır. Örneğin; çalışanların hataları, bilgisayar virüsleri ve

\footnotetext{
*Sorumlu yazar: mcibuk@beu.edu.tr

Geliş Tarihi: 24.02.2021, Kabul Tarihi: 29.04.2021
} 
hizmet engelleme (denial of service - DoS) saldırıları bunlardan bir kaçıdır. Yapılan saldırılar sonucu önemli bilgi kayıpları olmakta ve gizli kalması gereken bilgiler ifşa edilebilmektedir. İnternetteki güvenlik açıkları, insanları internete karşı güvensiz hale getirmekte ve web tabanlı şirketlere ve kamu hizmetlerine büyük zarar verebilmektedir. Bu yüzden şirketler ve kamu hizmetleri yürüten kurumlar, güvenlik tedbirlerini arttırmakta ve olası tehditlerin üstesinden gelmek amacıyla daha büyük yatırımlar yapmak zorunda kalmaktadırlar. Bundan dolayı, bilgisayar sistemlerinin güvenliğini sağlayan araçlar gittikçe önem kazanmakta, özellikle de Saldırı Tespit Sistemlerine (STS) duyulan önem her geçen gün artmaktadır. STS, ağ üzerinden yapılan her türlü saldırılara karşı bilişim sistemlerinin korunmasına yardımcı olup, uyarı niteliği taşıyan yazılım veya donanım bileşenlerinin tümüne denilmektedir [1]. STS kullanılarak ağ üzerinden yapılan saldırılar tespit edilebilmekte ve ilgili mekanizmalar harekete geçirilerek engellenebilmektedir. STS uygulamalarında makine öğrenmesi, veri madenciliği vb. farklı yöntemler kullanılsa da yapay zekâ tekniklerine dayalı yöntemler de sıkça kullanılmaya başlanmıştır. Literatürde saldırı tespiti için yapılan bazı çalışmaları şöyle sıralayabiliriz.

Burukanlı ve ark. [2] yaptıkları çalışmada makine öğrenme algoritmaları kullanarak saldırı tespiti gerçekleştirmiş̧lerdir. KDD'99 veri setinin \%100'lük kısmı üzerinde 5-kat çapraz doğrulama yapmışlardır. En iyi başarımı \%100 oranıyla Topluluk Öğrenme (TÖ) sınıflandırıcılarından olan torbalı ağaçlar (bagged trees) algoritması elde etmiştir. Sağıroğlu ve ark [1] yaptıkları çalışmada, Çok Katmalı Ağlar (ÇKA) tabanlı etkili bir STS geliştirmişlerdir. KDD'99 veri setinden 65536 örnek kullanmışlardır. Elde ettikleri en yüksek başarım oranı \% 97,92 ve en düşük başarım oranı ise \%81,93 olarak gerçekleşmiştir. Sonawane ve ark. [3] yaptıkları çalışmada, Yapay Sinir Ağı (YSA) ve Temel Bileşen Analizi (TBA) tabanlı ağ modeli kullanılarak saldırı tespiti gerçekleştirmişlerdir. Ayrıca, her iki algoritmanın kıyaslanması yapılmış olup, YSA'nın TBA'ya göre daha iyi sonuç verdiği gözlemlenmiştir. Aburomman ve ark. [4] yaptıkları çalışmada, TÖ sınıflandırıcısı kullanılarak saldırı tespiti uygulamalarına yeni bir boyut kazandırmışlardır. Çalışmalarında, en iyi başarım oranı \%85,17 olarak gerçekleşmiştir. Golovko ve ark. [5] çalışmalarında, TBA, Devirdaim Sinir Ağları ve ÇKA ağ modellerini kullanarak saldırı tespiti yapmışlardır. Model 1, Model 2 ve Model 3 olarak 3 tane model önermişlerdir. Çalışmalarında, en iyi performansı Model 3'ün DoS veri setinde \%99,9 olarak gerçekleştiğini gözlemlemişlerdir. Wang ve ark. [6] yaptıkları çalışmada, TBA tabanlı model kullanarak saldırı tespiti gerçekleştirmişlerdir. Çalışmada sonucunda, başarım oranı \%98,8 olarak gerçekleşmiştir. Sonawane ve ark. [7] yaptıkları çalışmada, TBA, Çekirdek TBA+Bayes tabanlı sinir ăg modelleri kullanarak saldırı tespiti gerçekleştirmişlerdir. TBA sinir ağ modeli ile Çekirdek TBA+Bayes Sinir A $\breve{g}$ Modelleri birbirleriyle kıyaslamışlardır. En iyi performansı \%92,70 ile Çekirdek TBA+Bayes Sinir A $\breve{g}$ Modeli elde etmiştir. Mukkamala ve ark. [8] yaptıkları çalışmada, sinir ağlar ve destek vektör makinelerini kullanarak saldırı tespitini gerçekleştirmişlerdir. Her iki öğrenme algoritması \%99'un üzerinde başarım elde etmiştir.

$\mathrm{Bu}$ çalışmada makine öğrenme yaklaşımlarının saldırı tespiti üzerindeki performansları araştırılmış olup. Tüm deneyler, açık erişime sunulmuş ve yaygın olarak kullanılan KDD'99 veri kümesi üzerinde gerçekleştirilmiştir. Sınıflandırıcı olarak, KA, TÖ ve DVM tercih edilmiştir. Sınıflandırma aşamasında 5-kat çapraz doğrulama tekniği kullanılmış olup, TÖ sınıflandırıcıların daha iyi başarım elde ettiği gözlemlenmiş̧tir.

\section{Materyal ve Metot}

\subsection{KDD'99 veri seti}

DARPA veri seti, ilk olarak Amerika Birleşik Devletlerinin Hava Kuvvetleri Ağı örnek alınarak tasarlanmış bir benzetim veri seti kümesidir [9]. DARPA tarafından desteklenen ilk çalışma Massachusetts Teknoloji Üniversitesi (MIT) tarafından 1998 y1lında gerçekleştirilmiştir.

DARPA veri seti bir takım ön işlemlerden geçirilerek KDD'99 veri seti elde edilmiştir. KDD'99, 9 temel özellik ve 32 adet türetilmiş özellik olmak üzere toplamda 41 adet özellikten oluşan bir veri setidir [10]. KDD'99 veri setinin kullanılmasının amacı; saldırı tespiti için eğitim ve test işlemleri bakımından kolaylık sağlamasıdır. Bu çalışmada kullanılan KDD10CORRECTED veri seti 494021 örnekten, KDDTEST veri seti 311029 örnekten ve bu iki veri setinin birleşiminden elde edilen KDD10CORRECTED+KDDTEST veri seti 805050 örnekten oluşmaktadır [10]. 
KDD10CORRECTED, KDDTEST ve KDD10CORRECTED+KDDTEST veri setlerinde bulunan saldırı tiplerinin miktarları ve yüzdelik oranları Tablo 1'de verilmiştir.

Tablo 1. KDD10CORRECTED, KDDTEST ve KDD10CORRECTED+KDDTEST veri setlerinde bulunan saldırı tiplerinin miktarları ve yüzdelik oranları $[10,11]$

\begin{tabular}{|c|c|c|c|c|c|c|}
\hline \multirow[b]{2}{*}{ Saldırı Tipi } & \multicolumn{2}{|c|}{ KDD10CORRECTED } & \multicolumn{2}{|c|}{ KDDTEST } & \multicolumn{2}{|c|}{ KDD10CORRECTED+KDDTEST } \\
\hline & Miktarı & $\begin{array}{l}\text { Yüzdelik } \\
\text { Oranı (\%) }\end{array}$ & Miktarı & $\begin{array}{c}\text { Yüzdelik } \\
\text { Oranı (\%) }\end{array}$ & Miktarı & $\begin{array}{c}\text { Yüzdelik Oranı } \\
(\%)\end{array}$ \\
\hline apache2 & - & - & 794 & 0,2552 & 794 & 0,0986 \\
\hline back & 2203 & 0,4459 & 1098 & 0,3530 & 3301 & 0,4100 \\
\hline buffer_overflow & 30 & 0,0060 & 22 & 0,0070 & 52 & 0,0064 \\
\hline ftp_write & 8 & 0,0016 & 3 & 0,0009 & 11 & 0,0013 \\
\hline guess_passwd & 53 & 0,0107 & 4367 & 1,4040 & 4420 & 0,5490 \\
\hline httptunnel & - & - & 158 & 0,0507 & 158 & 0,0196 \\
\hline imap & 12 & 0,0024 & 1 & 0,0003 & 13 & 0,0016 \\
\hline ipsweep & 1247 & 0,2524 & 306 & 0,0983 & 1553 & 0,1929 \\
\hline land & 21 & 0,0042 & 9 & 0,0028 & 30 & 0,0037 \\
\hline loadmodule & 9 & 0,0018 & 2 & 0,0006 & 11 & 0,0013 \\
\hline mailbomb & - & - & 5000 & 1,6075 & 5000 & 0,6210 \\
\hline mscan & - & - & 1053 & 0,3385 & 1053 & 0,1307 \\
\hline multihop & 7 & 0,0014 & 18 & 0,0057 & 25 & 0,0031 \\
\hline named & - & - & 17 & 0,0054 & 17 & 0,0021 \\
\hline neptune & 107201 & 21,6996 & 58001 & 18,6481 & 165202 & 20,5207 \\
\hline nmap & 231 & 0,0467 & 84 & 0,0270 & 315 & 0,0391 \\
\hline normal & 97278 & 19,6910 & 60593 & 19,4814 & 157871 & 19,6100 \\
\hline perl & 3 & 0,0006 & 2 & 0,0006 & 5 & 0,0006 \\
\hline phf & 4 & 0,0008 & 2 & 0,0006 & 6 & 0,0007 \\
\hline pod & 264 & 0,0534 & 87 & 0,0279 & 351 & 0,0435 \\
\hline portsweep & 1040 & 0,2105 & 354 & 0,1138 & 1394 & 0,1731 \\
\hline processtable & - & - & 759 & 0,2440 & 759 & 0,0942 \\
\hline ps & - & - & 16 & 0,0051 & 16 & 0,0019 \\
\hline rootkit & 10 & 0,0020 & 13 & 0,0041 & 23 & 0,0028 \\
\hline saint & - & - & 736 & 0,2366 & 736 & 0,0914 \\
\hline satan & 1589 & 0,3216 & 1633 & 0,5250 & 3222 & 0,4002 \\
\hline sendmail & - & - & 17 & 0,0054 & 17 & 0,0021 \\
\hline smurf & 280790 & 56,8376 & 164091 & 52,7574 & 444881 & 55,2612 \\
\hline snmpgetattack & - & - & 7741 & 2,4888 & 7741 & 0,9615 \\
\hline snmpguess & - & - & 2406 & 0,7735 & 2406 & 0,2988 \\
\hline spy & 2 & 0,0004 & - & - & 2 & 0,0002 \\
\hline sqlattack & - & - & 2 & 0,0006 & 2 & 0,0002 \\
\hline teardrop & 979 & 0,1981 & 12 & 0,0038 & 991 & 0,1230 \\
\hline udpstorm & - & - & 2 & 0,0006 & 2 & 0,0002 \\
\hline warezclient & 1020 & 0,2064 & - & - & 1020 & 0,1267 \\
\hline warezmaster & 20 & 0,0040 & 1602 & 0,5150 & 1622 & 0,2014 \\
\hline worm & - & - & 2 & 0,0006 & 2 & 0,0002 \\
\hline xlock & - & - & 9 & 0,0028 & 9 & 0,0011 \\
\hline xsnoop & - & - & 4 & 0,0012 & 4 & 0,0004 \\
\hline xterm & - & - & 13 & 0,0041 & 13 & 0,0016 \\
\hline Toplam & 494021 & 100 & 311029 & 100 & 805050 & 100 \\
\hline
\end{tabular}

Tablo 2'de KDD'99 veri setinin 41 özelliği ve bu özelliklerin tipleri ve Tablo 3'te ise KDD'99 veri setinin birkaç örneği gösterilmiştir. 
Tablo 2. KDD'99 veri setinin 41 özelliği ve bu özelliklerin tipleri [10, 11]

\begin{tabular}{|c|c|}
\hline Özellik Adı & Özellik Tipi \\
\hline duration & Sürekli \\
\hline protocol_type & Sembolik \\
\hline service & Sembolik \\
\hline flag & Sembolik \\
\hline src_bytes & Sürekli \\
\hline dst_bytes & Sürekli \\
\hline land & Sembolik \\
\hline wrong_fragment & Sürekli \\
\hline urgent & Sürekli \\
\hline hot & Sürekli \\
\hline num_failed_logins & Sürekli \\
\hline logged_in & Sembolik \\
\hline num_compromised & Sürekli \\
\hline root_shell & Sürekli \\
\hline su_attempted & Sürekli \\
\hline num_root & Sürekli \\
\hline num_file_creations & Sürekli \\
\hline num_shells & Sürekli \\
\hline num_access_files & Sürekli \\
\hline num_outbound_cmds & Sürekli \\
\hline is_host_login & Sembolik \\
\hline is_guest_login & Sembolik \\
\hline count & Sürekli \\
\hline srv_count & Sürekli \\
\hline serror_rate & Sürekli \\
\hline srv_serror_rate & Sürekli \\
\hline rerror_rate & Sürekli \\
\hline srv_rerror_rate & Sürekli \\
\hline same_srv_rate & Sürekli \\
\hline diff_srv_rate & Sürekli \\
\hline srv_diff_host_rate & Sürekli \\
\hline dst_host_count & Sürekli \\
\hline dst_host_srv_count & Sürekli \\
\hline dst_host_same_srv_rate & Sürekli \\
\hline dst_host_diff_srv_rate & Sürekli \\
\hline dst_host_same_src_port_rate & Sürekli \\
\hline dst_host_srv_diff_host_rate & Sürekli \\
\hline dst_host_serror_rate & Sürekli \\
\hline dst_host_srv_serror_rate & Sürekli \\
\hline dst_host_rerror_rate & Sürekli \\
\hline dst_host_srv_rerror_rate & Sürekli \\
\hline
\end{tabular}

Tablo 3. KDD'99 veri setinin birkaç saldırı tipi örneği [10]

\begin{tabular}{|c|c|}
\hline Saldırı Tipi & Örnek \\
\hline normal & 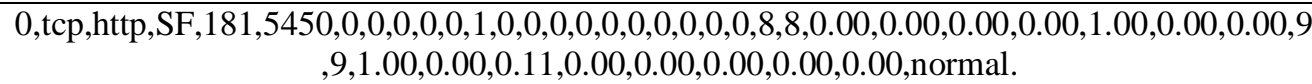 \\
\hline neptune & $\begin{array}{c}0, \text { tcp,private, } \mathrm{S} 0,0,0,0,0,0,0,0,0,0,0,0,0,0,0,0,0,0,0,201,1,1.00,1.00,0.00,0.00,0.00,0.06,0.00,2 \\
55,1,0.00,0.08,0.00,0.00,1.00,1.00,0.00,0.00 \text {, neptune }\end{array}$ \\
\hline warezclient & $\begin{array}{c}1, \mathrm{tcp}, \mathrm{ftp}, \mathrm{SF}, 1267,2451,0,0,0,28,0,1,0,0,0,0,0,0,0,0,0,1,1,1,0.00,0.00,0.00,0.00,1.00,0.00,0.00, \\
110,8,0.07,0.05,0.01,0.00,0.02,0.00,0.07,0.00, \text { warezclient }\end{array}$ \\
\hline satan & $\begin{array}{c}0, \text { udp,private, SF } 1,0,0,0,0,0,0,0,0,0,0,0,0,0,0,0,0,0,13,1,0.00,0.00,0.00,0.00,0.08,0.69,0.00,25 \\
5,1,0.00,0.26,1.00,0.00,0.00,0.00,0.00,0.00 \text {,satan }\end{array}$ \\
\hline
\end{tabular}




\subsection{Temel Bileșen Analizi}

Temel Bileşen Analizi (TBA), bilgisayar bilimlerinde boyut azaltmak için kullanılan bir yöntemdir [12]. TBA, sinyal işleme, görüntü işleme ve yapay zekâ tekniklerinde boyut azaltmak için sıkça kullanılan tekniklerden bir tanesidir [11]. TBA, var olan verinin daha az sayıda boyutla ifade etmesi, fazla öneme sahip olmayan boyutların çıkarılması ve önemli olan boyutların kullanılması olayı olarak da ifade edilebilir [11, 13].

Bir giriş dizi vektörü $X_{1}, X_{2}, X_{3}, \ldots, X_{n} \in R^{n}(n<m)$ ve $\sum_{t=1}^{m} X_{t}=0$ ise [11, 14], vektörlerin kovaryans matrisi denklem (1)'deki gibi hesaplanır:

$$
\begin{aligned}
& C=\frac{1}{m} \sum_{t=1}^{m} X t X t^{T} \\
& \lambda_{t} U_{t}=C U_{t}
\end{aligned}
$$

Burada; $\chi_{t}, U_{t}, k$ ve $\theta$, sirasıyla $C$ kovaryasının öz değeri, öz vektörlerin özdeşi, öz vektörlerin en büyüğü ve eşik değeri en büyük $\mathrm{k}$ öz vektörünün yaklaşık duyarlılı̆̆ını ifade eder [11, 14].

$$
\sum_{t=1}^{k} \lambda_{t} \backslash \sum_{t=1}^{m} \lambda_{t} \geq \theta
$$

$\theta$,eşik değerine bakılarak;

$$
\begin{aligned}
& U=\left[U_{1}, U_{2}, U_{3}, \ldots, U_{k}\right] \\
& \Lambda=\left[\chi_{1}, \lambda_{2}, \chi_{3}, \ldots, \chi_{k}\right] \\
& S=U^{T} X_{t}
\end{aligned}
$$

Elde edilen yeni boyut vektörü denklem (6)'daki gibi hesaplanır [11, 14].

\subsection{Karar Ăgacı}

Karar Ağaçları (KA), sınıflandırma ve tahmin için sıklıkla başvurulan bir öğrenme yaklaşımıdır. Düşük maliyetli olması, anlaşılması ve yorumlanması kolay olması bakımından sıklıkla tercih edilmektedir.

KA'larında belirsizlik ve kararsızlık önemli bir sorundur bunun üstesinden gelmek için en yaygın olarak kullanılan entropi ölçümü kullanılmaktadır. Entropi ölçümü ne kadar fazla ise çıkan sonuçlar da o oranda belirsiz ve kararsız olmaktadır. Bu yüzden, KA'larında entropi ölçüsü en az olan alanlar tercih edilmektedir $[2,11,15,16]$.

Entropi değeri denklem (7)'deki gibi hesaplanır [11, 15].

$\operatorname{Entropi}(S)=\sum_{i}^{C}-p_{i} \log p_{i}$

Burada;

$C$, hedef özellikteki değer sayısı (sınıfların sayısı) veya bir özelliğe atanan maksimum değer sayısı $p_{i}$, i sinıfındaki örneklerin sayısı

Bir özelliğin bilgi kazancı denklem (8)'deki gibi hesaplanır [11].

$\operatorname{Kazanç}(S, A)=\operatorname{Entropi}(S)-\sum_{V \in(A)} \frac{\left|S_{V}\right|}{S} \operatorname{Entropi}\left(S_{V}\right)$ 
Burada; $A$ : Özellik, $V: A$ özelliğinin bir olası değeri, $S_{V}: \mathrm{V}$ değeri için örneklerin sayısı, $S$ : tüm veri örneklerinin sayısıdır [11].

\subsection{Topluluk Öğrenme}

Topluluk Öğrenme (TÖ), birçok öğrenme algoritmasının bir arada kullanılmasıdır [2, 11]. Diğer bir deyişle; TÖ, birçok zayıf öğrenme algoritmasının birleştirilerek daha güçlü ve daha iyi sonuçlar elde eden bir öğrenme algoritmasının oluşturulması yöntemidir [2, 11]. Tek bir algoritmaya göre daha iyi sonuç vermektedir. TÖ, en iyi sınıflandırma yöntemlerinden biridir [17]. Ayrıca genelleme yetenekleri oldukça gü̈çlüdür. TÖ, sınıflandırma ve regresyon için sıkça kullanılan öğrenme yöntemidir. En yaygın kullanılan TÖ teknikleri Bagging ve Boosting'tır [2, 11].

\subsection{Destek Vektör Makinesi}

Destek Vektör Makinesi (DVM), sınıflandırma ve regresyon problemlerinin çözümü için sıkça kullanılan bir denetimli öğrenme yöntemidir [2]. DVM, oldukça yüksek genelleme yapabilme yeteneğine sahiptir.

DVM'nin en önemli avantajı yüksek oranda başarılı sonuçlar elde etmeleridir. En önemli dezavantajı ise çok geç sonuç vermeleridir. DVM, margini maksimum yapan bir en uygun ayırıcı düzlemi oluşturmaya çalışmaktadır. Örneğin, bir örnek uzayında eğitim örnekleri denklem (9)'daki formülle birbirinde ayırılabilmektedir [11].

$$
f(x)=w \cdot x+b=0
$$

Doğrusal olmayan durumlarda çekirdek adı verilen fonksiyonlar kullanılmaktadır. Sınıflandırma yapılırken yüksek boyutlu uzaya taşınan vektörler doğrusal olarak ayrılmaktadır. Ayrılan düzlemler içerisinde sınıflara uzaklığı en fazla olan doğrusal ayrıştırıcı olarak belirlenmektedir. Yüzeye en yakın vektörler belirlenerek en yakın uzaklık tespit edilir.

$K=\left\{\left(x_{1}, y_{1}\right),\left(x_{2}, y_{2}\right), \ldots,\left(x_{N}, y_{N}\right)\right\}$ bir eğitim seti olarak verilsin. $i=1,2, \ldots, N . \quad x_{i} \in R^{n}$ ve $y_{i} \in$ $\{-1,1\}$.

K'nın optimal ayrılabilir bir hiper düzlemi $(f(x)=w \cdot x+b=0)$ olarak tanımlanabilir [11]. Burada;

$f(x)=\left(w_{0} \cdot x\right)+b_{0}$

$w_{0}=\sum_{j=1}^{N} y_{j} \alpha_{j}^{0} x_{j}$

$w_{0}=\left(w_{0}^{1}, w_{0}^{2}, \ldots, w_{0}^{n}\right)$ ve $x=\left(x^{1}, x^{2}, \ldots, x^{n}\right)$ olarak verilmiş olsun. Bu durumda bu iki vektörün iç çarpımı denklem (12)'de gösterilmiştir.

$$
\begin{aligned}
& \left(w_{0} \cdot x\right)=\sum_{i=1}^{n} w_{0}^{i} \cdot x^{i} \\
& b_{0}=y_{i}-\left(x_{i} \cdot \sum_{j=1}^{N} y_{j} \alpha_{j}^{0} x_{j}\right)
\end{aligned}
$$

Denklem (11)'deki $w_{0}$, denklem (10)'da yerine yazılırsa denklem (14) elde edilir. 
$f(x)=\sum_{i=1}^{N} y_{i} \alpha_{i}^{0}\left(x_{i} \cdot x\right)+b_{0}$

$y_{i}\left(w_{0} \cdot x_{i}-b\right) \geq 1, i=1,2, \ldots, N$.

Denklem (15)'teki optimal hiper düzlem karar fonksiyonu kullanarak iki sınıfın birbirinden doğrusal olarak ayrılıp ayrılmadığı kontrol edilebilir[8, 11, 18-21].

$f(x)=\operatorname{sgn}\left(w \cdot x_{i}+b\right)=\operatorname{sgn}\left(\sum_{i=1}^{N} \alpha_{i} y_{i}\left(x_{i} \cdot x\right)+b\right)$

\section{Bulgular ve Tartışma}

Bu çalışmada on iki farklı sınıflandırma algoritması kullanılarak KDD10CORRECTED, KDDTEST ve KDD10CORRECTED+KDDTEST veri setleri üzerinde, her bir sınıflandırıcının başarım değerleri elde edilmiştir. KDD10CORRECTED ve KDDTEST veri setleri, 4 çekirdekli Intel Core i5-4590S işlemci, 4 GB Ram ve Intel HD Graphics 4600 bilgisayarda eğitilmiştir.

KDD10CORRECTED+KDDTEST veri seti ise veri miktarının fazla olmasından dolayı Intel Xeon E5620 ( 2 işlemci 8 çekirdek), 16 GB Ram ve NVIDIA Quadro K2000 ekran kartı olan iş istasyonunda eğitilmiştir. Tüm deneyler MATLAB ortamında sınıflandırma öğrenme aracı kullanılarak gerçekleştirilmiştir. Her bir sınıflandırma yönteminin varsayılan özellikleri kullanılmıştır. Tablo 4'te TÖ sınıflandırıcıların temel özellikleri, Tablo 5'te DVM sınıflandırıcıların temel özellikleri ve Tablo 6'da ise KA sınıflandırıcıların temel özellikleri gösterilmiştir.

Tablo 4. TÖ sınıflandırıcıların temel özellikleri

\begin{tabular}{|c|c|c|c|c|c|c|}
\hline $\begin{array}{c}\text { Ana } \\
\text { Teknik }\end{array}$ & $\begin{array}{c}\text { Kullanılan } \\
\text { Teknik }\end{array}$ & TBA & $\begin{array}{c}\text { Öğrenici } \\
\text { Tipi }\end{array}$ & $\begin{array}{c}\text { Maksimum } \\
\text { Bölme Sayısı }\end{array}$ & $\begin{array}{c}\text { Öğrenici } \\
\text { Sayısı }\end{array}$ & $\begin{array}{c}\text { Öğrenme } \\
\text { Oranı }\end{array}$ \\
\hline TÖ & $\begin{array}{l}\text { Uyarlamalı } \\
\text { Güçlendirme }\end{array}$ & $\begin{array}{l}\text { Pasif/ } \\
\text { Aktif }\end{array}$ & $\begin{array}{l}\text { Karar } \\
\text { Ağacı }\end{array}$ & 20 & 30 & 0.1 \\
\hline TÖ & Torbalama & $\begin{array}{l}\text { Pasif/ } \\
\text { Aktif }\end{array}$ & $\begin{array}{l}\text { Karar } \\
\text { Ağacı }\end{array}$ & - & 30 & - \\
\hline TÖ & $\begin{array}{c}\text { Rastgele Alt } \\
\text { Örnekleme } \\
\text { Artırma }\end{array}$ & $\begin{array}{l}\text { Pasif/ } \\
\text { Aktif }\end{array}$ & $\begin{array}{l}\text { Karar } \\
\text { Ağacı }\end{array}$ & 20 & 30 & 0.1 \\
\hline
\end{tabular}

Tablo 5. DVM sinıflandırıcıların temel özellikleri

\begin{tabular}{cccccccc}
\hline $\begin{array}{c}\text { Ana } \\
\text { Teknik }\end{array}$ & $\begin{array}{c}\text { Kullanılan } \\
\text { Teknik }\end{array}$ & TBA & $\begin{array}{c}\text { Çekirdek } \\
\text { Fonksiyonu }\end{array}$ & $\begin{array}{c}\text { Çekirdek } \\
\text { Ölçeği }\end{array}$ & $\begin{array}{c}\text { Kutu } \\
\text { Kısıtlama } \\
\text { Seviyesi }\end{array}$ & $\begin{array}{c}\text { Çoklu } \\
\text { Sınıf } \\
\text { Metodu }\end{array}$ & $\begin{array}{c}\text { Verileri } \\
\text { Standartlaştırma }\end{array}$ \\
\hline DVM & Doğrusal DVM & $\begin{array}{c}\text { Pasif/ } \\
\text { Aktif }\end{array}$ & Doğrusal & Otomatik & 1 & $\begin{array}{c}\text { Bire } \\
\text { Karş1 Bir } \\
\text { Bire }\end{array}$ & Evet \\
DVM & Karesel DVM & $\begin{array}{c}\text { Pasif/ } \\
\text { Aktif }\end{array}$ & Karesel & Otomatik & 1 & $\begin{array}{c}\text { Karş1 Bir } \\
\text { Bire }\end{array}$ & Evet \\
DVM & Kübik DVM & $\begin{array}{c}\text { Pasif/ } \\
\text { Aktif }\end{array}$ & Kübik & Otomatik & 1 & $\begin{array}{c}\text { Karş1 Bir } \\
\text { Bire }\end{array}$ & Evet \\
DVM & $\begin{array}{c}\text { Hassas Gauss } \\
\text { DVM }\end{array}$ & $\begin{array}{c}\text { Pasif/ } \\
\text { Aktif }\end{array}$ & Gauss & 1.6 & 1 & $\begin{array}{c}\text { Karş1 Bir } \\
\text { Bire }\end{array}$ & Evet \\
DVM & $\begin{array}{c}\text { Ortalama Gauss } \\
\text { DVM }\end{array}$ & $\begin{array}{c}\text { Pasif/ } \\
\text { Aktif }\end{array}$ & Gauss & 6.4 & 1 & $\begin{array}{c}\text { Karş1 Bir } \\
\text { Bire } \\
\text { Karş1 Bir }\end{array}$ & Evet \\
DVM & $\begin{array}{c}\text { Kaba Gauss } \\
\text { DVM }\end{array}$ & $\begin{array}{c}\text { Pasif/ } \\
\text { Aktif }\end{array}$ & Gauss & 26 & 1 & & Evet \\
\hline
\end{tabular}


Tablo 6. KA sınıflandırıcıların temel özellikleri

\begin{tabular}{cccccc}
\hline Ana Teknik & $\begin{array}{c}\text { Kullanılan } \\
\text { Teknik }\end{array}$ & TBA & $\begin{array}{c}\text { Maksimum } \\
\text { Bölme Sayısı }\end{array}$ & Bölme Kriteri & $\begin{array}{c}\text { Vekil Karar } \\
\text { Bölmeleri }\end{array}$ \\
\hline KA & Hassas Ağaç & Pasif/Aktif & 100 & $\begin{array}{c}\text { Gini’nin } \\
\text { Çeşitlilik } \\
\text { İndeksi }\end{array}$ & Pasif \\
KA & Ortalama Ağaç & Pasif/Aktif & 20 & $\begin{array}{c}\text { Gini’nin } \\
\text { Çeşitlilik } \\
\text { İndeksi }\end{array}$ & Pasif \\
& Kaba Ağaç & Pasif/Aktif & 4 & Gini’nin & Çeşitlilik \\
KA & & & İndeksi & Pasif \\
\hline
\end{tabular}

Bu çalışmada kullanılan on iki adet sınıflandırıcının değerlendirme metrikleri için Tablo 7'de hata matrisi kullanılarak elde edilmiştir.

Tablo 7. Hata matrisi [11]

\begin{tabular}{|c|c|c|c|}
\hline \multicolumn{2}{|c|}{ Hata Matrisi } & \multicolumn{2}{c|}{ Tahmin Edilen Sınıf } \\
\cline { 2 - 4 } & & Pozitif (Saldırı) & Negatif (Normal) \\
\hline \multirow{2}{*}{ Gerçek Sınıf } & Pozitif (Saldırı) & Doğru Pozitif (DP) & Yanlış Negatif (YN) \\
\cline { 2 - 4 } & Negatif (Normal) & Yanlış Pozitif(YP) & Doğru Negatif (DN) \\
\hline
\end{tabular}

Doğru Pozitif (DP): Gerçekte pozitif (saldırı) olan ve tahmin edildiğinde de pozitif (saldırı) olarak sinıflandırılan örnekleri ifade etmektedir.

Yanlış Negatif (YN): Gerçekte pozitif (saldırı) olan ve tahmin edildiğinde de negatif (normal) olarak siniflandırılan örnekleri ifade etmektedir.

Yanlış Pozitif (YP): Gerçekte negatif (normal) olan ve tahmin edildiğinde de pozitif (saldırı) olarak sinıflandırılan örnekleri ifade etmektedir.

Doğru Negatif (DN): Gerçekte negatif (normal) olan ve tahmin edildiğinde de negatif (normal) olarak sınıflandırılan örnekleri ifade etmektedir. Denklem (16)'da bu çalışmada kullanılan başarım ölçütü verilmiştir $[2,11,22]$.

$\operatorname{Doğruluk}($ Accuracy $)=\frac{D P+D N}{D P+Y N+Y P+D N}$

Tablo 8'de KDD10CORRECTED veri seti üzerinde 12 adet sinıflandırıcının performans karşılaştırılması, Tablo 9'da KDDTEST veri seti üzerinde 12 adet sınıflandırıcının performans karşılaştırılması ve Tablo 10'da ise KDD10CORRECTED+KDDTEST veri seti üzerinde 12 adet sınıflandırıcının performans karşılaştırılması gösterilmiştir.

Tablo 8. KDD10CORRECTED veri seti üzerinde 12 adet sınıflandırıııın performans karşılaştırılması

\begin{tabular}{lcccc}
\hline & \multicolumn{2}{c}{ TBA Pasif } & \multicolumn{2}{c}{ TBA Aktif } \\
\hline Sınıflandırıcılar & Doğruluk & $\begin{array}{c}\text { Ĕ̆itim Süresi } \\
(\mathbf{s n})\end{array}$ & Doğruluk & $\begin{array}{c}\text { Ĕgitim } \\
\text { Süresi (sn) }\end{array}$ \\
\hline Hassas Ağaç & $\% 99,90$ & 831,58 & $\% 99,90$ & 762,08 \\
Ortalama Ağaç & $\% 99,50$ & 1028,9 & $\% 99,50$ & 744,09 \\
Kaba Ağaç & $\% 98,30$ & 894,34 & $\% 98,20$ & 687,03 \\
Uyarlamalı Güçlendirme (Adaboost) & $\% 99,80$ & 636,4 & $\% 99,60$ & 598,14 \\
Torbalama (Bagging) & $\mathbf{\% 9 9 , 9 9}$ & $\mathbf{4 4 6 , 6 8}$ & $\mathbf{\% 9 9 , 9 0}$ & $\mathbf{3 6 8 , 7 3}$ \\
Rastgele Alt Örnekleme Artırma (RUSBoost) & $\% 91,80$ & 399,99 & $\% 84,70$ & 359,64 \\
Doğrusal DVM & $\% 99,90$ & 2769 & $\% 89,60$ & 12228 \\
Karesel DVM & $\% 99,90$ & 6919,4 & $\% 82,20$ & 65404 \\
Kübik DVM & $\% 86,30$ & 62429 & $\% 86,80$ & 130300 \\
Hassas Gauss DVM & $\% 99,80$ & 15394 & $\% 99,40$ & 1783,3 \\
Ortalama Gauss DVM & $\% 99,90$ & 4177,8 & $\% 99,40$ & 1807 \\
Kaba Gauss DVM & $\% 99,90$ & 2917,6 & $\% 99,20$ & 2628,5 \\
\hline
\end{tabular}


Yapılan çalışma sonucunda Tablo 8'de göründüğü gibi, 12 sınıflandırıcı arasında TBA pasif durumda iken en iyi performansı TÖ sınıflandırıcısından olan \% 099,99 ile Torbalama sınıflandırıcısı elde etmiştir. En kötü performansı ise DVM sınıflandırıcısından olan \%86,30 ile Kübik DVM sınıflandırıcıs1 elde etmiştir. Öte yandan, TBA aktif durumda iken en iyi performansları TÖ sınıflandırıcısından olan \%99,90 ile Torbalama sınıflandırıcısı ile KA sınıflandırıcısından olan \%99,90 ile Hassas Ağaç elde etmiştir. Torbalama sınıflandırıcısı ile Hassas Ağaç sınıflandırıcısı aynı başarım oranına sahip olmasına rağmen, eğitim ve test süresi bakımından en iyi sonucu yaklaşık 369 sn ile Torbalama sınıflandırıcısı elde etmiştir. En kötü performansı ise DVM sınıflandırıcısından olan \%82,2 ile Karesel DVM sinıflandırıcısı elde etmiştir. KDD10CORRECTED veri seti üzerinde 12 adet sinıflandırıcının performanslarının karşılaştırılması konunun daha iyi anlaşılması için Şekil 1'de grafiksel olarak da gösterilmiştir.

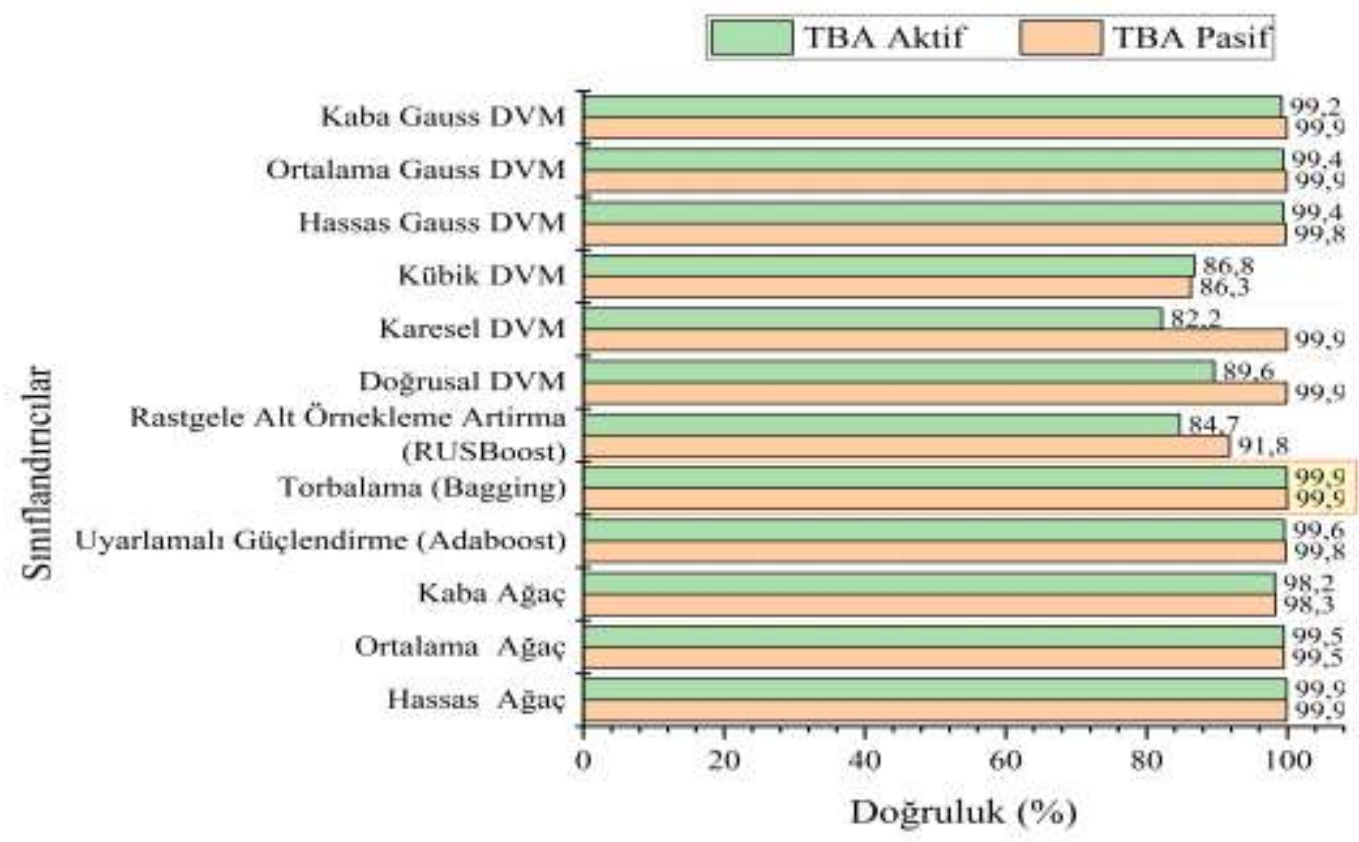

Şekil 1. KDD10CORRECTED veri seti üzerinde 12 adet sınıflandırıcının performans karşılaştırılması

Tablo 9. KDDTEST veri seti üzerinde 12 adet sınıflandırıcının performans karşılaştırılması

\begin{tabular}{lcccc}
\hline & \multicolumn{2}{c}{ TBA Pasif } & \multicolumn{2}{c}{ TBA Aktif } \\
\hline Sinıflandırıcılar & Doğruluk & $\begin{array}{c}\text { Ĕgitim } \\
\text { Süresi (sn) }\end{array}$ & Doğruluk & $\begin{array}{c}\text { Ĕgitim } \\
\text { Süresi (sn) }\end{array}$ \\
\hline Hassas Ağaç & $\% 97,30$ & 22,822 & $\% 96,80$ & 30,893 \\
Ortalama Ağaç & $\% 95,10$ & 13,347 & $\% 94,90$ & 22,018 \\
Kaba Ağaç & $\% 90,90$ & 10,544 & $\% 90,90$ & 22,015 \\
Uyarlamalı Güçlendirme (Adaboost) & $\% 96,50$ & 599,6 & $\% 95,80$ & 480,98 \\
Torbalama (Bagging) & $\mathbf{9 9 7 , 9 0}$ & $\mathbf{2 9 6 , 7 3}$ & $\mathbf{\% 9 7 , 6 0}$ & $\mathbf{2 3 6 , 5 5}$ \\
Rastgele Alt Örnekleme Artırma (RUSBoost) & $\% 73,00$ & 376,24 & $\% 55,20$ & 367,3 \\
Doğrusal DVM & $\% 96,30$ & 9507,7 & $\% 95,60$ & 14826 \\
Karesel DVM & $\% 96,90$ & 16756 & $\% 77,00$ & 25586 \\
Kübik DVM & $\% 78,90$ & 125060 & $\% 71,10$ & 74122 \\
Hassas Gauss DVM & $\% 96,60$ & 15306 & $\% 95,80$ & 5308,9 \\
Ortalama Gauss DVM & $\% 96,30$ & 13022 & $\% 95,70$ & 5519,9 \\
Kaba Gauss DVM & $\% 96,30$ & 10493 & $\% 95,30$ & 8058 \\
\hline
\end{tabular}

Yapılan çalışma sonucunda Tablo 9'da göründüğü gibi, 12 sınıflandırıcı arasında TBA pasif durumda iken en iyi performansı TÖ sınıflandırıcısından olan \%97,9 ile Torbalama sinıflandırıcısı elde etmiştir. En kötü performansı ise TÖ sınıflandırıcısından olan \%73 ile Rastgele Alt Örnekleme Artırma 
sinıflandırıcısı elde etmiştir. Öte yandan, TBA aktif durumda iken en iyi performansı TÖ sınıflandırıcısından olan \%97,60 ile Torbalama sınıflandırıcısı elde etmiştir. En kötü performansı ise DVM sınıflandırıcısından olan \%71,10 ile Kübik DVM sınıflandırıcısı elde etmiştir. KDDTEST veri seti üzerinde 12 adet sınıflandırıcının performanslarının karşılaştırılması konunun daha iyi anlaşılması için Şekil 2'de grafiksel olarak da gösterilmiştir.

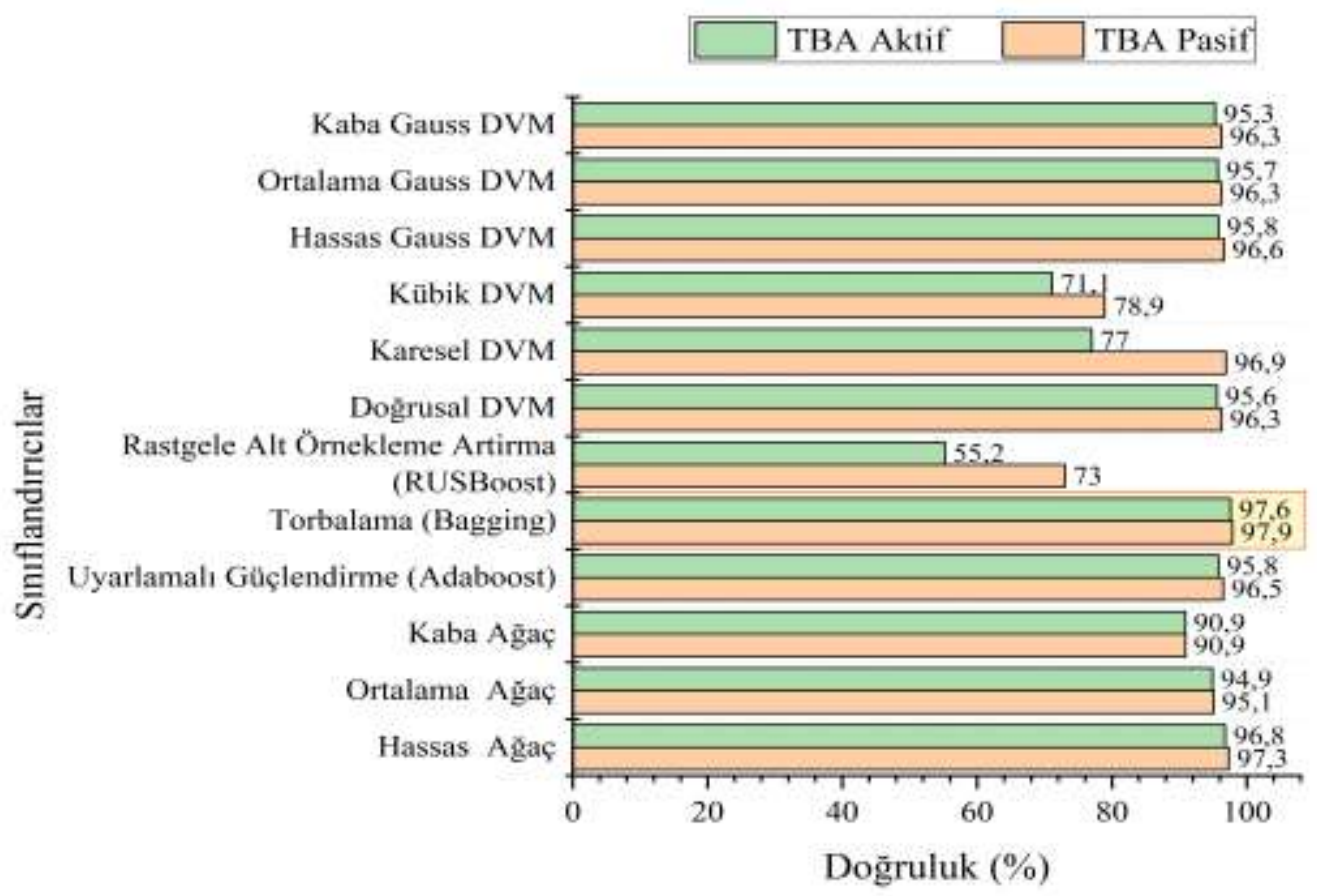

Şekil 2. KDDTEST veri seti üzerinde 12 adet sınıflandırıcının performans karşılaştııılması

Tablo 10. KDD10CORRECTED+KDDTEST veri seti üzerinde 12 adet sinıflandırıcının performans karşılaştırılması

\begin{tabular}{lcccc}
\hline & \multicolumn{2}{c}{ TBA Pasif } & \multicolumn{2}{c}{ TBA Aktif } \\
\hline Sinıflandırıcılar & Doğruluk & $\begin{array}{c}\text { Ĕgitim } \\
\text { Süresi (sn) }\end{array}$ & Doğruluk & $\begin{array}{c}\text { Ĕ̆itim } \\
\text { Süresi (sn) }\end{array}$ \\
\hline Hassas Ağaç & $\% 98,40$ & 62,736 & $\% 98,30$ & 100,72 \\
Ortalama Ağaç & $\% 97,00$ & 43,566 & $\% 96,80$ & 61,132 \\
Kaba Ağaç & $\% 95,20$ & 37,23 & $\% 94,90$ & 75,452 \\
Uyarlamalı Güçlendirme (Adaboost) & $\% 98,20$ & 2080,5 & $\% 98,10$ & 1612 \\
Torbalama (Bagging) & $\mathbf{\% 1 0 0 , 0 0}$ & $\mathbf{1 1 9 1 , 5}$ & $\mathbf{\% 9 8 , 8 0}$ & $\mathbf{9 5 1 , 7 6}$ \\
Rastgele Alt Örnekleme Artırma (RUSBoost) & $\% 80,90$ & 1298,6 & $\% 65,00$ & 1211,1 \\
Doğrusal DVM & $\% 98,50$ & 11720 & $\% 81,10$ & 57348 \\
Karesel DVM & $\% 98,50$ & 89777 & $\% 76,80$ & 273860 \\
Kübik DVM & $\% 75,60$ & 668140 & $\% 72,60$ & 486570 \\
Hassas Gauss DVM & $\% 98,60$ & 75506 & $\% 97,30$ & 8522,3 \\
Ortalama Gauss DVM & $\% 98,50$ & 20729 & $\% 97,20$ & 8861 \\
Kaba Gauss DVM & $\% 98,50$ & 13795 & $\% 97,00$ & 13070 \\
\hline
\end{tabular}

Yapılan çalışma sonucunda Tablo 10'da göründüğü gibi, 12 sınıflandırıcı arasında TBA pasif durumda iken en iyi performansı TÖ sinıflandırıcısından olan \%100 ile Torbalama sınıflandırıcısı elde etmiştir. En kötü performansı ise DVM sınıflandırıcısından olan \%75,60 ile Kübik DVM sınıflandırıcısı elde etmiştir. Öte yandan, TBA aktif durumda iken en iyi performansı TÖ sınıflandırıcısından olan $\% 98,80$ ile Torbalama sınıflandırıcısı elde etmiştir. En kötü performansı ise TÖ sınıflandırıcısından olan $\% 65,00$ ile Rastgele Alt Örnekleme Artırma sınıflandırıcısı elde etmiştir. KDD10CORRECTED+KDDTEST veri seti üzerinde 12 adet siniflandırıcının performanslarının karşılaştırılması konunun daha iyi anlaşılması için Şekil 3'de grafiksel olarak da gösterilmiştir. 


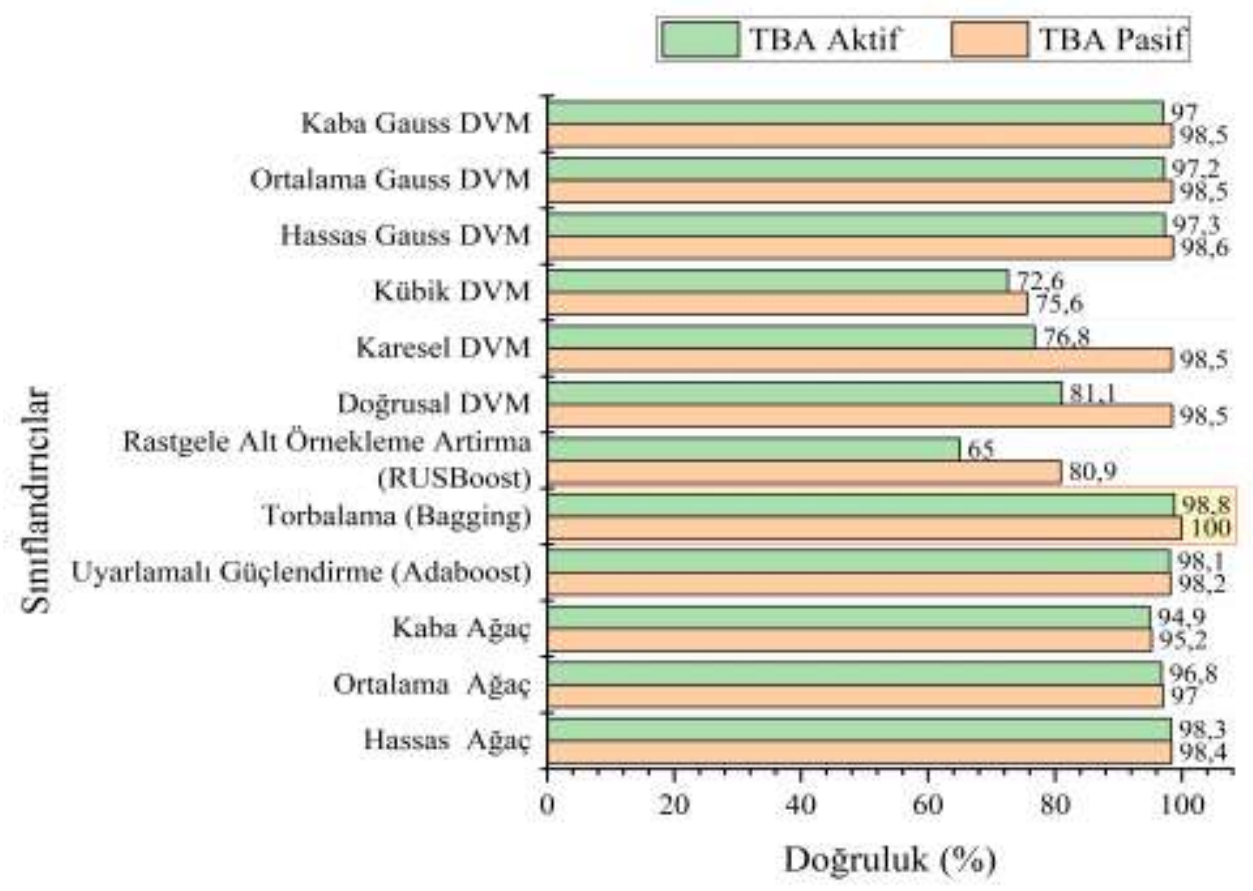

Şekil 3. KDD10CORRECTED+KDDTEST veri seti üzerinde 12 adet sınıflandırıcının performans karşılaştırılması

\section{Sonuç ve Öneriler}

Bu çalışmada, günümüzde en çok kullanılan makine öğrenme sınıflandırıcıları kullanılarak saldırı tespiti gerçekleştirilmiştir. Makine öğrenme sınıflandırıcıları olarak; KA, TÖ ve DVM sınıflandırıcıları tercih edilmiştir. $\mathrm{Bu}$ üç sınıflandırma tekniği kullanılarak KDD'99 veri kümesi altındaki bulunan; KDD10CORRECTED, KDDTEST ve KDD10CORRECTED+KDDTEST veri seti üzerinde sınıflandırma işlemi gerçekleştirilmiştir. Sınıflandırma aşamasında 5-kat çapraz doğrulama tekniği kullanılmış olup, en iyi başarım oranlarına Tablo 8, Tablo 9 ve Tablo 10 incelendiğinde KDD10CORRECTED veri setinde üzerinde $\% 99,99$, KDDTEST veri setinde üzerinde $\% 97,90$ ve KDD10CORRECTED+KDDTEST veri setinde üzerinde \%100 ile Torbalama sinıflandırıcis1 sahip olmuştur. Bunun nedeni olarak TÖ sınıflandırıcıların birden fazla iyi başarım gösteren algoritmaları birleştirilerek başarım oranını mümkün olduğunca yükseltilmesi olarak düşünülebilir. Sonuç olarak bu tarz çalışmalar için TÖ sınıflandırıcıları, KA ve DVM'ye göre daha iyi başarım elde ettiği görülmüştür. $\mathrm{Bu}$ veri setleri için TÖ sınıflandırıcılarından olan Torbalama sınıflandırıcısının tercih edilmesi daha yerinde olacaktır.

\section{Yazarların Katkısı}

Tüm yazarlar eşit oranda katkı sağlamıştır.

\section{Çıkar Çatışması Beyanı}

Yazarlar arasında herhangi bir çıkar çatışması bulunmamaktadır.

\section{Araştırma ve Yayın Etiği Beyanı}

Yapılan çalışmada araştırma ve yayın etiğine uyulmuştur.

\section{Kaynaklar}

[1] Sağıroğlu Ş., Yolaçan E.N., Yavanoğlu U. 2011. Zeki Saldırı Tespit Sistemi Tasarımı ve Gerçekleştirilmesi. J Fac Eng Arch Gazi Univ, 26 (2): 325-340. 
[2] Burukanlı M., Budak Ü., Çıbuk M. 2019. Saldırı Tespit Sistemlerinde Makine Öğrenme Metotlarının Kullanımı. In: Uluslararası Bilim ve Mühendislik Sempozyumu, 20-22 Haziran 2019, Siirt, Türkiye, 1052-1057.

[3] Sonawane H.A., Pattewar T.M. 2015. A Comparative Performance Evaluation of Intrusion Detection Based on Neural Network and PCA. In: 2015 Int. Conf. Commun. Signal Process. ICCSP 2015, 841-845.

[4] Aburomman A.A. Reaz M.B.I. 2017. A Survey of Intrusion Detection Systems Based on Ensemble and Hybrid Classifiers. Comput Secur, 65: 135-152.

[5] Golovko V.A., Vaitsekhovich L.U., Kochurko P.A., Rubanau U.S. 2007. Dimensionality Reduction and Attack Recognition Using Neural Network Approaches. In: 2007 Int. Jt. Conf. Neural Networks. IEEE, 12-17 Aug. 2007, Orlando, FL, USA, 2734-2739.

[6] Wang W., Battiti R. 2006. Identifying Intrusions in Computer Networks with Principal Component Analysis. In: First Int. Conf. Availability, Reliab. Secur. IEEE, 20-22 April 2006, Vienna, Austria, Austria, 270-279.

[7] Pattewar T.M., Sonawane H.A. 2015. Neural Network Based Intrusion Detection Using Bayesian with PCA and KPCA Feature Extraction. In: 2015 IEEE Int. Conf. Comput. Graph. Vis. Inf. Secur. IEEE, 2-3 Nov. 2015, Bhubaneswar, India, 83-88.

[8] Mukkamala S., Janoski G., Sung A. 2002. Intrusion Detection Using Neural Networks and Support Vector Machines. In: Proc. 2002 Int. Jt. Conf. Neural Networks. IJCNN'02 (Cat. No.02CH37290). IEEE, 12-17 May 2002, Honolulu, HI, USA, USA, 1702-1707.

[9] Özgür A., Erdem H. 2012. Saldırı Tespit Sistemlerinde Kullanılan Kolay Erişilen Makine Öğrenme Algoritmalarının Karşılaştırılması. Biliş̧im Teknol Derg., 5 (2): 41-48.

[10] Anonim, 1999. The UCI KDD Archive Information and Computer Science University of California, Irvine. http://kdd.ics.uci.edu/databases/kddcup99/kddcup99.html (Erişim Tarihi: 26.01.2021).

[11] Burukanlı M. 2020. Copula Fonksiyonlarını Kullanarak Bilgisayar Ağlarında Saldırı Tespiti. Yüksek Lisans Tezi, Bitlis Eren Üniversitesi Lisansüstü Eğitim Enstitüsü, Bitlis.

[12] Liu W.M., Chang C.I. 2007. Variants of Principal Components Analysis. In: Int. Geosci. Remote Sens. Symp., 1083-1086.

[13] Abdi H., Williams L.J. 2010. Principal Component Analysis. Wiley Interdiscip Rev Comput Stat, 2 (4): 433-459.

[14] Jinhu L., Xuemei L., Lixing D., Liangzhong J. 2010. Applying Principal Component Analysis and Weighted Support Vector Machine in Building Cooling Load Forecasting. In: 2010 Int. Conf. Comput. Commun. Technol. Agric. Eng. 12-13 June 2010, Chengdu, China, 434-437.

[15] Çalış A., Kayapınar S., Çetinyokuş T. 2014. Veri Madenciliğinde Karar Ağaci Algoritmalari ile Bilgisayar ve İnternet Güvenliği Üzerine Bir Uygulama. Endüstri Mühendisliği Derg., 25 (3-4): 2-19.

[16] Safavian S.R., Landgrebe D. 1991. A Survey of Decision Tree Classifier Methodology. IEEE Trans Syst Man Cybern, 21 (3): 660-674.

[17] Yu Y., Zhong Liang F., Xiang Hui Z., Wen Fang C. 2009. Combining Classifier Based on Decision Tree. In: 2009 WASE Int. Conf. Inf. Eng. IEEE, 10-11 July 2009, Taiyuan, Chanxi, China, 37-40.

[18] Masud M., Khan L., Thuraisingham B. 2011. Data Mining Tools for Malware Detection. CRC Press. Taylor \& Francis, London.

[19] Lu S-X., Meng J., Cao G-E. 2010. Support Vector Machine Based on A New Reduced Samples Method. In: 2010 Int. Conf. Mach. Learn. Cybern. IEEE, 11-14 July 2010, Qingdao, China, 15101514.

[20] Kim H.C., Pang S., Je H.M., Kim D., Bang S.Y. 2003. Constructing Support Vector Machine Ensemble. Pattern Recognit, 36 (12): 2757-2767.

[21] Crbuk M., Budak U., Guo Y., Cevdet Ince M., Sengur A., 2019. Efficient Deep Features Selections and Classification for Flower Species Recognition. Meas J Int Meas Confed, 137: 713.

[22] Budak Ü., Cömert Z., Çıbuk M., Şengür A. 2020. DCCMED-Net: Densely Connected and Concatenated Multi Encoder-Decoder CNNs for Retinal Vessel Extraction from Fundus Images. Med Hypotheses, 134: 1-9. 